\title{
Adaptación y validación de un cuestionario para valorar la motivación en el contexto deportivo
}

\author{
Adaptation and validation of a questionnaire to \\ assess motivation in the sport context
}

\section{Adaptação e validação de um questionário para avaliar a motivação no contexto desportivo}

Juan José Pulido González, David Sánchez-Oliva ${ }^{1}$, Inmaculada González-Ponce ${ }^{1}$, Diana Amado Alonso ${ }^{1}$, Carlos Montero Carretero ${ }^{2}$ y Tomás García-Calvo ${ }^{1}$

${ }^{1}$ Universidad de Extremadura $y^{2}$ Universidad Miguel Hernández.

Resumen: El objetivo de este estudio era adaptar y validar al contexto deportivo el Cuestionario de Motivación en Educación Física $(C M E F)$, basado en la Teoría de la Autodeterminación (Deci y Ryan, 1985; 2000; Ryan y Deci, 2000) y desarrollado originalmente en el ámbito educativo, para tratar de analizar la motivación intrínseca, identificada, introyectada, externa y desmotivación de los adolescentes. Para ello, participaron un total de 985 deportistas con edades comprendidas entre los 10 y 16 años $(M=14.34 ; D T$ $=2.52$ ), de género masculino y femenino pertenecientes a 24 modalidades deportivas diferentes. Los resultados obtenidos demostraron que la escala con cinco factores correlacionados presentaba una adecuada validez factorial a partir de los índices de ajuste obtenidos, registró relaciones positivas entre los motivos de práctica más autodeterminados y relaciones negativas con la desmotivación, que oscilaron de entre -,21 a ,74. Además, se obtuvieron valores aceptables de consistencia interna $(\Omega>.70)$ y resultó invariante en función del género, considerando las mínimas modificaciones sufridas en $\triangle$ CFI. Asimismo, la validez discriminante de los factores resultó satisfactoria. Por tanto, este estudio aporta una nueva herramienta que permite analizar los tipos de regulación motivacional que impulsan a los adolescentes a realizar una práctica deportiva.

Palabras clave: adolescentes, autodeterminación, deporte, escala.

Abstract: The aim of this study was to adapt and validate into the sport context the Motivational Questionnaire in Physical Education (CMEF), based on Self-Determination Theory (Deci \& Ryan, 1985; 2000; Ryan \& Deci, 2000) and developed to be used in the educative domain, to assess the intrinsic motivation, identified, introyected, external and amotivation. Thus, 985 athletes, ranging in age from 10 to 16 years old $(M=14.34 ; S D$ $=2.52$ ), both male and female belonged to 24 different sport modalities, participated in the study. Results showed that the scale with five factors correlated had an adequate factorial validity through the fit index, with positive relationships between types of motivation more self-determined and negative relations with the amotivation, ranging from - ,21 to ,74. In addition, acceptable internal consistency values $(\Omega>.70)$ were obtained and an invariance analysis taking into account the minimum modifications obtained in $\triangle \mathrm{CFI}$ was conducted. Besides, the discriminant validity of the factors was satisfactory. Therefore, this research provides a new instrument that allows examining the types of motivational regulation that the adolescents have to participate in sport.

Keywords: adolescents, self-determination, sport, scale.

Resumo: O objetivo deste estudo era adaptar e validar no contexto desportivo o Questionário de Motivação em Educação Física (CMEF), com base na Teoria da Autodeterminação (Deci \& Ryan, 1985; 2000; Ryan \& Deci, 2000) e desenvolvido originalmente para ser utilizado no âmbito educativo, para analisar a motivação intrínseca, identificada, introjetada, externa e amotivação de adolescentes. Para ele, participaram um total de 985 despor tistas com idades compreendidas entre 10 e 16 anos $(M=14.34 ; D P=2.52)$, do género masculino e feminino pertencentes a 24 diferentes modalidades desportivas. Os resultados obtidos demonstraram que a escala com cinco factores correlacionados apresentava uma adequada validez factorial a partir dos índices de ajuste obtidos, com relaçôes positivas entre razôes de práticas mais auto-determinada e relaçôes negativas com amotivação, que vão desde - ,21 a ,74. Além disso, valores de consistência interna aceitáveis $(\Omega>$ .70) foram obtidos e provou gênero invariável, tendo em conta as mínimas modificaçōes sofridas no Confirmatory Fit Index ( $\triangle \mathrm{CFI})$. Asimismo, validade discriminante dos fatores foi satisfatória. Portanto o estudo contribuiu para uma nova ferramenta que permite analisar os tipos de regulaçáo motivacional que impulsam os adolescentes a realizar uma prática desportiva. Palavras chave: Adolescentes, autodeterminação, desporto, escala.

\section{Introducción}

Son diferentes los motivos que llevan a un sujeto a practicar algún tipo de actividad física, demostrándose que según sean Dirección para correspondencia [Correspondence address]: Juan José
Pulido González. Facultad de Ciencias del Deporte. Universidad de
Extremadura. Avenida de la Universidad, s/n. 10071. Cáceres (Espana). E-mail: jjpulido@unex.es estos determinantes, derivan en la aparición de consecuencias positivas o negativas (Vallerand, 2007). En este sentido, multitud de estudios se han apoyado en la Teoría de Autodeterminación (TAD: Deci y Ryan, 1985; 2000; Ryan y Deci, 2000) con el propósito de explicar las regulaciones motivacionales que impulsan a una persona a desarrollar una determinada 
actividad desde diferentes contextos, como el académico (Barkoukis y Hagger, 2013), el deportivo (Hendry, Crocker, y Hodges, 2014) o el laboral (Moran, Russinova, Yim, y Sprague, 2013). Estas regulaciones pueden estar dirigidas desde una interiorización hacia la práctica o pueden venir desencadenadas por motivos extrínsecos, lo que da lugar a un continuo motivacional que fluctúa en diferentes grados de autodeterminación.

Tradicionalmente, los postulados de la TAD diferenciaban tres grandes bloques motivacionales: la motivación intrínseca, la motivación extrínseca y la desmotivación. En primer lugar, la motivación intrínseca se caracteriza porque la persona siente placer y satisfacción por el mero hecho de realizar una actividad. Seguidamente, en un segundo nivel se encuentra la motivación extrínseca, subdividida a su vez en diferentes regulaciones enumeradas de mayor a menor autodeterminación según el continuo motivacional. Siguiendo este orden, se encuentra la regulación integrada, definida porque la actividad está inmersa en el estilo de vida de la persona, en la que se ponen de manifiesto características que tienen que ver con los valores, las metas, las necesidades personales, la identidad, la dieta, cuidados sanitarios, etc. A continuación, la regulación identificada se define por los beneficios que el individuo encuentra en aquello que realiza. Posteriormente, la regulación introyectada se caracteriza por el sentimiento de culpa que aparece en el caso de no haber participado, o por la realización de la actividad por orgullo. Seguidamente, la regulación externa hace referencia a la participación por una recompensa o premio. Por último, la desmotivación se define como la total ausencia de regulaciones intrínsecas y extrínsecas en la que el individuo no encontraría el sentido de seguir practicando la actividad que realiza.

Sin embargo, las últimas aportaciones a la TAD (Vansteenkiste, Lens, y Deci, 2006; Vansteenkiste, Niemiec, y Soenens, 2010), principalmente sustentadas en las altas correlaciones encontradas entre las regulaciones intrínseca e identificada, se decantan más por una agrupación formada por la motivación autónoma (compuesta por la motivación intrínseca y la regulación identificada), motivación controlada (formada por las regulaciones externa e identificada) y desmotivación. De esta manera, las personas con un alto grado de orientación autónoma tienden a actuar de acuerdo con sus principios y acordes con sus intereses, interpretando los acontecimientos externos como información y no como fin y, por lo tanto, soliendo regular su conducta de manera autónoma. Por el contrario, aparecen individuos con una orientación de control, los cuales tienden a actuar de acuerdo con una demanda externa, de forma presionada u ordenada desde el exterior y, por consiguiente, su regulación de comportamiento viene influenciada y determinada por una experiencia de control. Por último, las personas en las que su prioridad se basa en la orientación impersonal, tienden a per- cibir sus experiencias de vida como más allá de algo propio y, en consecuencia, son propensos al sentimiento omnipresente de impotencia, inoperancia y pasividad (Vansteenkiste et al., 2010). Esta orientación es equivalente a una disposición de desmotivación y se relaciona conceptualmente con el concepto de locus de control externo (Rotter, 1966).

En base a todo lo anterior, durante las últimas décadas se han diseńado diferentes instrumentos encaminados a analizar el tipo de regulación motivacional de una persona hacia la práctica deportiva. Generalmente, se ha utilizado la Sport Motivation Scale (SMS: Pelletier et al., 1995), desarrollada originariamente en francés (EMS: Échelle de motivation dans les Sports; Brière, Vallerand, Blais, y Pelletier, 1995) traducida y validada al castellano por diferentes estudios (Balaguer, Castillo, y Duda, 2007; Guzmán, Carratalá, García-Ferriol, y Carratalá, 2010; López, 2000; Núñez, Martín-Albo, y Navarro, 2007). Este instrumento está compuesto por 28 ítems, los cuales subdividen la motivación intrínseca en tres subescalas (al conocimiento, al logro y a las experiencias estimulantes), conformando un total de siete factores para analizar la autodeterminación de los deportistas. Sin embargo, este instrumento ha presentado ciertas limitaciones, derivadas de la traducción y su posterior aplicación con muestras con características culturales, educativas y socio-económicas diferentes.

Concretamente, Mallett, Kawabata y Newcombe (2007) ante la falta de validez convergente y discriminante, afirmaban la necesidad de volver a desarrollar la SMS, abordando una nueva forma a la hora de examinar la motivación intrínseca, extrínseca y desmotivación, así como también contemplando el análisis de la regulación integrada. Valorando estos posibles problemas de aplicabilidad y limitaciones en las propiedades psicométricas (Mallett, Kawabata, Newcombe, Otero-Forero, y Jackson, 2007; Martin y Cutler, 2002; Pelletier et al., 1995; Pelletier, Vallerand, y Sarrazin, 2007; Vlachopoulos, Karageorghis, y Terry, 2000), los propios autores de este instrumento y los autores que postularon originariamente la TAD (Pelletier, Rocchi, Vallerand, Deci, y Ryan, 2013) llevaron a cabo una segunda versión de la escala (SMS - II) para examinar la validez de constructo y la fiabilidad del cuestionario. Los resultados mostraron que la nueva escala se comportaba mejor que la versión anterior, mostrándose válida y fiable, aunque concluyen e insisten en mejorar las formas de medida para potenciar futuras investigaciones.

Paralelamente, Lonsdale, Hodge y Rose (2008) elaboraron un instrumento similar creado en función de los problemas psicométricos encontrados en la $S M S$ para valorar la regulación motivacional en el deporte (BRSQ: Behavioral Regulation in Sport Questionnaire), traducido y validado al castellano por varios autores (Moreno, Marzo, Martínez-Galindo, y Marín, 2011; Viladrich, Torregrosa, y Cruz, 2011). Esta escala está compuesta por un total de 24 ítems que analizan las regulaciones intrínseca, integrada, identificada, introyectada, 
externa y la desmotivación. Las novedades de este instrumento es que contempla a la motivación intrínseca como un constructo general e incluye la regulación integrada, la cual no se valoraba mediante la SMS. Además, un reciente estudio realizado por Lonsdale, Hodge, Hargreaves y Ng (2014) trataba de examinar las fortalezas y debilidades mediante la comparación de la $S M S$ - II y el $B R S Q$, con el propósito de establecer unas pautas y direcciones a la hora de valorar la motivación en el contexto deportivo. Las conclusiones del estudio informaban que ambas escalas mostraron una adecuada validez de constructo en las puntuaciones derivadas de las dos medidas, pero que no se encontraba suficiente información para afirmar que una escala fuera más adecuada que la otra.

En este sentido, a pesar de que existen diversos instrumentos diseñados y validados para conocer la motivación de los deportistas, éstos han presentado ciertos problemas de aplicabilidad. En las adaptaciones del BRSQ al castellano (Moreno et al., 2011; Viladrich et al., 2011) existen limitaciones en cuanto a la consistencia interna de los factores. Concretamente, en los estudios de Moreno et al. (2011) y Viladrich et al. (2011), las regulaciones identificada y externa no superaron el .70 (Nunnally, 1978). En base a estos resultados, y teniendo en cuenta los resultados positivos encontrados en las versiones originales de estas escalas, en cuanto a la consistencia interna, parece posible que el hecho de traducir estos instrumentos para ser utilizados en un contexto diferente, con adolescentes bajo condiciones culturales también distintas, podría provocar la pérdida de calidad psicométrica en el análisis de la motivación deportiva.

Ante esta controversia y limitaciones de las dos escalas más importantes desarrolladas para valorar la motivación en el deporte en el contexto español, uno de los principales objetivos de este estudio era adaptar y validar al deporte el Cuestionario de Motivación del contexto de la Educación Física (SánchezOliva, Leo, Amado, González-Ponce, y García-Calvo, 2012), proporcionando un instrumento específico para el ámbito deportivo que analice fiablemente las regulaciones motivacionales de los jóvenes deportistas. De la misma manera, se pretendía comprobar la invarianza de la escala considerando para ello el género de los participantes. Derivado de la principal finalidad de la investigación, las hipótesis que guiaron la misma son que el $C M E F$ adaptado al contexto deportivo mostraría una adecuada propiedad psicométrica en términos de validez y fiabilidad. Asimismo, el instrumento resultaría invariante en función del género de los deportistas.

\section{Método}

\section{Participantes}

En el desarrollo de la investigación participaron un total de 985 deportistas federados en el programa a nivel regional de- nominado JUDEX (Juegos Deportivos de Extremadura), con edades comprendidas entre los 10 y 16 años $(M=14,34 ; D T$ $=2,52$ ). Del conjunto de participantes, 620 deportistas eran de género masculino y 365 de género femenino, de deportes individuales $(n=452)$ y colectivos $(n=533)$ de 24 disciplinas diferentes. Se utilizó una metodología correlacional y el proceso de selección de la muestra fue intencionado, considerando para ello la distribución geográfica de los clubes y las posibilidades de desplazamiento de los investigadores, así como se tuvo en cuenta las categorías de los equipos y el género de los participantes para disponer de una muestra más heterogénea. Del total de cuestionarios cumplimentados, se decidió eliminar el 6,34\%, ya que se presentaban incompletos, con la misma puntuación otorgada en todos los ítems, contestados en sitios dónde no se debía hacer, etc.

\section{Instrumentos}

Regulación motivacional de los deportistas. Para conocer el tipo de regulación motivacional de los deportistas se ha utilizado el Cuestionario de Motivación en el Deporte (CMD) que se valida en este estudio. Se inicia con la frase: "Yo practico este deporte por...", acompañado de 20 ítems que valoran la motivación intrínseca ( 4 ítems, ejemplo: "Porque me lo paso bien realizando este deporte"), la motivación extrínseca; regulación identificada (4 ítems, ejemplo: "Porque puedo aprender habilidades que podrían ser útiles en otras áreas de mi vida"), introyectada (4 ítems, ejemplo: "Porque me siento mal conmigo mismo si no lo hago") y externa (4 ítems, ejemplo: "Porque está bien visto por el entrenador y los compañeros"), y la desmotivación (4 ítems, ejemplo: "Realmente siento que estoy perdiendo mi tiempo haciendo este deporte"). Considerando la explicación que da el estudio de Vallerand y Rosseau (2001), que afirman que la regulación integrada no es característica en nińos y adolescentes, puesto que en estas etapas no llegan a integrarse plenamente los diferentes aspectos que configuran el estilo de vida y la personalidad, no se ha incluido el análisis de este tipo de regulación motivacional en este instrumento. El formato de respuesta empleado fue mediante una escala tipo Likert en la que cada ítem tenía un rango de 1 a 5 . El 1 se correspondía con totalmente en desacuerdo y el 5 con totalmente de acuerdo con el ítem formulado.

\section{Procedimiento}

En primer lugar, la investigación fue aprobada por la Comisión Ética de la Universidad de Extremadura, cumpliendo las normas establecidas en la Declaración de Helsinki de 1964. Seguidamente, se estableció un protocolo de actuación para que la recogida de datos fuera similar en todos los participantes, ya que previamente el investigador principal se puso 
en contacto con las distintas federaciones para explicarles el objetivo del estudio. Una vez que los clubes dieron su visto bueno, se les explicó a los entrenadores la idiosincrasia del estudio y se les facilitó un consentimiento informado que recaería en los padres de los deportistas para autorizar a sus hijos como participantes del estudio, puesto que éstos eran en su totalidad menores de edad. Posteriormente, también fueron informados los propios deportistas de las finalidades de la investigación y de la confidencialidad en el tratamiento y difusión de los datos obtenidos, cumpliendo con las exigencias éticas que marca la American Psychological Association (APA, 2009). De esta forma, los deportistas cumplimentaron los cuestionarios justo antes de empezar la sesión de entrenamiento. Para ello, se contó con la ayuda de uno de los investigadores, que asistió para estar presente en la recogida de datos y prestar la atención necesaria ante cualquier duda o imprevisto por parte de los adolescentes. Por último, el proceso de realización tuvo una duración aproximada de entre 10-15 minutos.

En cuanto al proceso de adaptación del instrumento, fue desarrollado por tres expertos en la temática de la investigación. De esta manera, cada uno de los investigadores, de manera individual, realizó una versión personalizada de lo que sería su adaptación. Posteriormente, compartieron sus ideas para llevar a cabo una propuesta común y definitiva. Los cambios se centraron en la composición de cada ítem, con la finalidad de elaborar un instrumento específico para el contexto deportivo, sustituyendo y completando cada constructo en cada caso. Por ejemplo, se modificó el significativo al que se refería la escala original: "mi profesor" por "mi entrenador". También sufrió cambios las palabras referentes al ámbito en el que se desarrolla el estudio: "clases de Educación Física" por "deporte, entrenamientos, sesiones, etc.". Asimismo, se modificaron los términos destinados a los protagonistas de la investigación: "alumnos" por "deportistas". Por último, se mantuvo la estructura psicométrica del instrumento, con el mismo número de ítems y factores que en su versión de referencia.

\section{Análisis de los datos}

Para el registro y análisis de datos se utilizaron los programas estadísticos SPSS 21,0 y Amos 19,0. Del mismo modo, con el objetivo de valorar las propiedades psicométricas de la escala, se llevó a cabo un Análisis Factorial Confirmatorio (AFC) con el Método de Estimación de Parámetros de Máxima Verosimilitud, utilizando los siguientes índices para verificar el ajuste del modelo: $\chi^{2} / \mathrm{gl}$ (Chi-Cuadrado / Grados de libertad), SRMR (Standardized Root Mean Residual), RMSEA (Root Mean Square Error of Approximation), CFI (Comparative Fit Index), TLI (Tucker-Lewis Index) y GFI (Goodness of Fit In$d e x)$. Además, se analizaron los estadísticos descriptivos, los valores de asimetría y curtosis y los índices de fiabilidad (alfa de Cronbach) de cada uno de los factores que componen el instrumento. Por último, se realizó un análisis de la invarianza del modelo en función del género para comprobar si resultaba invariante según este parámetro.

\section{Resultados}

\section{Análisis de la validez factorial}

Para analizar la validez factorial de la adaptación del $C M E F$ al deporte, se realizó un AFC de la estructura de cinco factores correlacionados (Figura 1) con el método de estimación de máxima verosimilitud y el procedimiento bootstrapping (coeficiente de Mardia = 82,46) siguiendo los indicadores recomendados por Byrne (2008), lo que aseguraba la robustez de los datos a pesar de la no normalidad de los mismos.

En el análisis se introdujeron como reactivos a los 20 ítems que conformaban la escala, agrupados en cinco factores de primer orden: regulación intrínseca, regulación identificada, regulación introyectada, regulación externa y desmotivación. Los datos obtenidos, reflejaron los siguientes índices: $\chi^{2} / \mathrm{gl}=$ 4,804; CFI = ,919; TLI = ,904; GFI = ,923; SRMR = ,049 y RMSEA = ,062. Según Hu y Bentler (1999) valores de CFI superiores a ,95 junto con valores iguales o inferiores de ,06 para RMSEA y ,08 para SRMR, indican un excelente ajuste del modelo. Sin embargo, muchos autores expertos en psicometría defienden que estos rangos son muy difícil de lograr al tratarse de modelos complejos que utilizan datos reales, en lugar de valores simulados (Marsh, Hau, y Grayson, 2005), por lo que se suelen considerar válidos valores superiores a ,90.

De la misma manera, en la Figura 1 se muestran los pesos de regresión de los ítems sobre cada uno de los factores (entre $, 52$ y, $82 ; p<.05)$, registrando además valores satisfactorios en las varianzas del error (entre $, 27 \mathrm{y}, 65$ ). Por último, los coeficientes de correlación entre los diferentes factores oscilaron entre,- 21 y, 74 . 
Figura 1. Modelo del Análisis Factorial Confirmatorio de la adaptación del CMEF con cinco factores correlacionados.

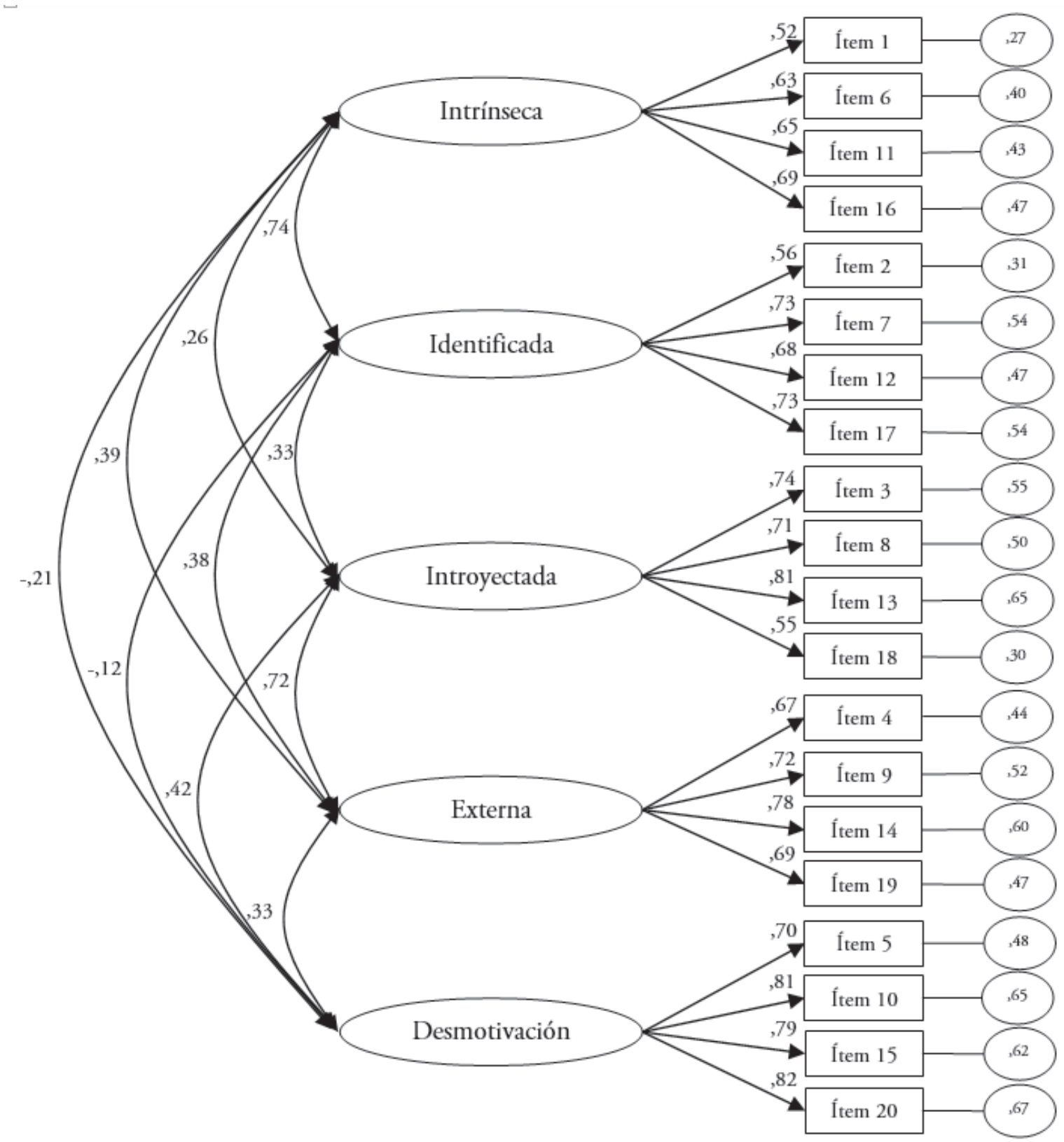

Seguidamente, se decidió testar un modelo de tres factores correlacionados de segundo orden, siguiendo una estructura de motivación autónoma que engloba dos factores de primer orden como son la motivación intrínseca e identificada, motivación controlada junto con otros dos factores de primer orden: motivación introyectada y externa y, desmotivación. De este modo, los resultados obtenidos mostraron los siguientes índices de ajuste: $\chi^{2} / \mathrm{gl}=4,847$; CFI $=, 917$; TLI $=, 903$; GFI $=, 920 ; \mathrm{SRMR}=, 052$ y RMSEA $=, 063$.
En este sentido, en la Figura 2 los pesos de regresión obtenidos de los ítems sobre cada uno de los factores oscilaron de entre ,52 y ,82 $(p<.05)$, con valores en las varianzas del error de entre, 27 y ,67. En cuanto a los coeficientes de correlación, las puntuaciones registradas entre los factores de segundo orden oscilaron entre -,19 y ,46 y entre ,84 y ,89 entre los factores de primer orden. 
Figura 2. Análisis Factorial Confirmatorio de la adaptación del CMEF con tres factores correlacionados.

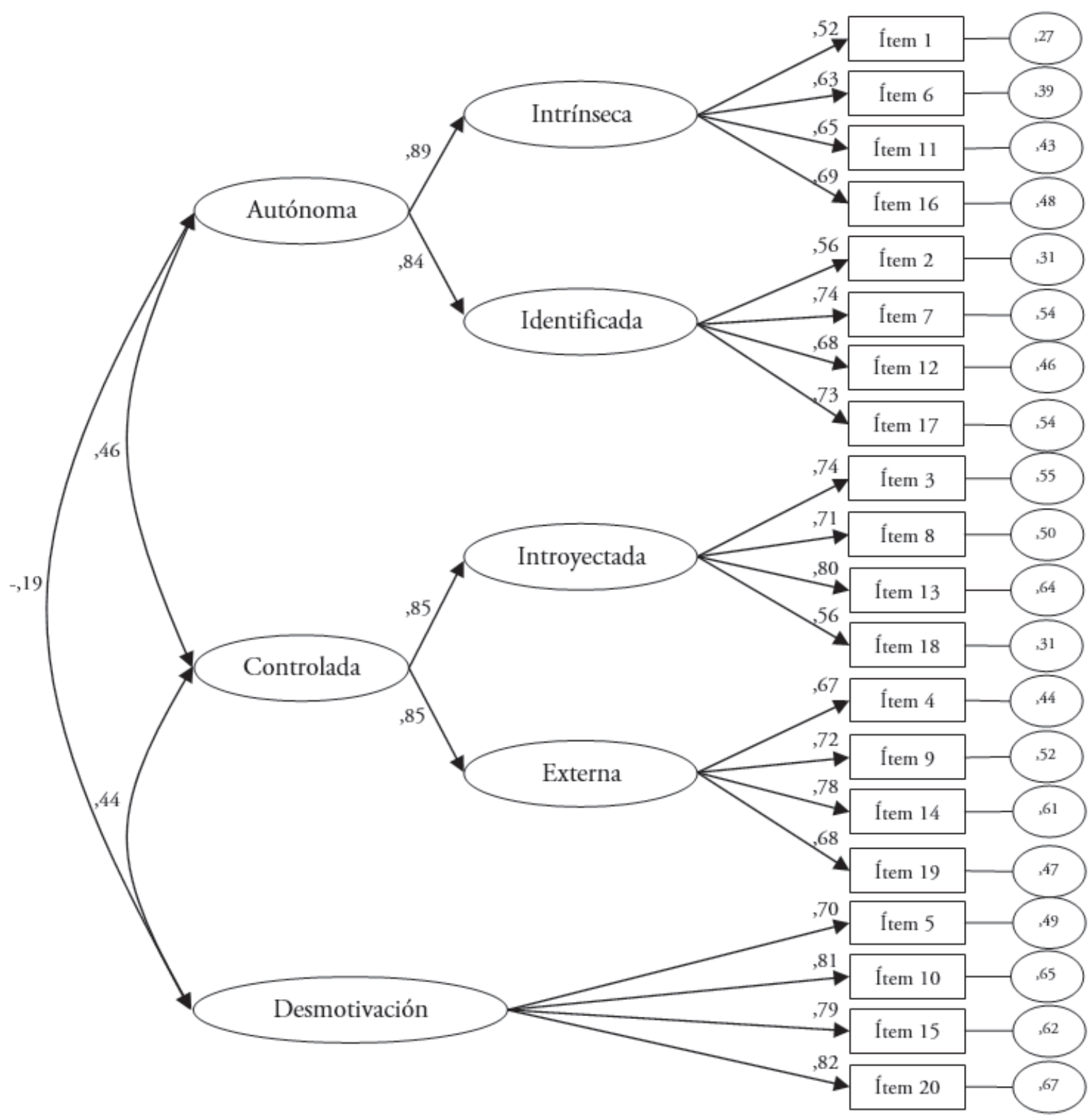

Análisis descriptivo, consistencia interna y validez discriminante

En la Tabla 1 se pueden observar los valores medios obtenidos en el instrumento de la adaptación del CMEF. De esta forma, la motivación intrínseca mostró la puntuación más elevada, descendiendo las puntuaciones en consonancia con los niveles de autodeterminación, excepto en la regulación externa, que obtuvo valores superiores respecto a la regulación introyectada. Asimismo, se muestra la consistencia interna de todas las variables (McDonald, 1985; 1999), con una confiabilidad de constructo (coeficiente omega) aceptable por encima de ,70 (Nunnally y Bernstein, 1994), oscilando específicamente desde, 72 a ,85. 
Tabla 1. Estadísticos descriptivos y fiabilidad de los factores de la adaptación del $C M E F$.

\begin{tabular}{lccccc}
\hline & $M$ & $D T$ & Asimetria & Curtosis & $\Omega$ \\
\hline Intrínseca & 4,54 &, 59 & $-1,74$ & 3,35 &, 72 \\
Identificada & 4,28 &, 71 & $-1,16$ & 1,30 &, 77 \\
Introyectada & 2,99 & 1,17 &, 03 &,- 91 &, 80 \\
Externa & 3,26 & 1,12 &,- 21 &,- 80 &, 80 \\
Desmotivación & 1,50 &, 92 & 2,33 & 4,96 &, 85 \\
\hline
\end{tabular}

En cuanto a la validez discriminante, se siguió el método de Bagozzi y Kimmel (1995), obteniendo resultados coherentes y satisfactorios. Para lograr la validez discriminante, la diferencia entre la unidad y el valor de la correlación debe exceder de 1,96 multiplicado por el error estándar de la correlación (Bagozzi y Kimmel, 1995). Esta condición se dio en todos los factores que componen el instrumento.

\section{Validez nomológica}

Para determinar la validez nomológica del instrumento, se ha llevado a cabo un análisis de correlaciones bivariadas entre dos de los factores que componen el Cuestionario de Motivación en el Deporte (intrínseca y desmotivación) y los tres factores de la Escala de Necesidades Psicológicas Básicas en el Ejercicio (BPNES; Sánchez y Núnéz, 2007). Como se aprecia en la Tabla 2, la motivación intrínseca se relacionó positivamente con las tres necesidades, destacando las asociaciones obtenidas con la necesidad de competencia. Asimismo, la desmotivación se relacionó negativamente con la competencia y con las relaciones sociales, mientras que no se relacionó con la autonomía.

Tabla 2. Correlaciones bivariadas entre los factores del CMD y la versión española de la Escala de Necesidades Psicológicas Básicas en el Ejercicio (BPNES).

\begin{tabular}{|c|c|c|c|}
\hline Variables & Autonomía & Competencia & Relaciones Sociales \\
\hline Intrínseca & ,379** &, $477^{* *}$ &, $405^{* *}$ \\
\hline Desmotivación & 054 &,$- 088^{* *}$ &,$- 117^{* *}$ \\
\hline
\end{tabular}

\section{Análisis de la invarianza}

Se analizó la invarianza de la estructura factorial en función del género, tratando de comprobar que no hubiera diferencias significativas considerando dicha variable. Como se aprecia en la Tabla 3, se tomó como referencia el modelo propuesto de cinco factores correlacionados, en el que se encontraron diferencias significativas entre chi cuadrado entre el modelo sin restricciones (Modelo 1) y los diferentes modelos con invarianza. Sin embargo, puesto que el coeficiente chi cuadrado es sensible al tamaño muestral, se empleó el criterio establecido por Cheung y Rensvold (2002) respecto al $\Delta$ CFI. Según estos autores, valores de $\Delta$ CFI menores o iguales a -,01 indican que no se puede rechazar la hipótesis nula de la invarianza. Por tanto, las puntuaciones registradas en este estudio respecto al $\triangle \mathrm{CFI}$ sugieren que la estructura factorial de la adaptación del $C M E F$ es invariante en función del género.

Tabla 3. Análisis de invarianza por género. Modelo con cinco factores correlacionados.

\begin{tabular}{lcccccccccc}
\hline & $\chi^{2}$ & $G l$ & $\chi^{2} / g l$ & $\Delta \mathrm{gl}$ & $\Delta \chi^{2}$ & $p$ & CFI & IFI & RMSEA & SRMR \\
\hline Modelo 1 & 949,250 & 320 & 2,966 & - & - & - &, 920 & .921 &, 045 &, 087 \\
Modelo 2 & 986,968 & 335 & 2,946 & 15 & 37,718 &, $001^{* *}$ &, 917 & .918 &, 044 &, 087 \\
Modelo 3 & 1003,453 & 350 & 2,867 & 15 & 16,485 &, 351 &, 917 & .918 &, 044 &, 090 \\
Modelo 4 & 1069,952 & 370 & 2,892 & 20 & 66,499 &, $008^{* *}$ &, 911 & .912 &, 044 &, 091 \\
\hline
\end{tabular}

Notas: Modelo 1 = Modelo sin restricciones; Modelo 2 = Modelo con pesos de medida; Modelo $3=$ Modelo con covarianzas estructurales; Modelo $4=$ Modelo con residuos de medida.

\section{Discusión}

Teniendo en cuenta el objetivo del trabajo que era adaptar y validar el Cuestionario de Motivación en Educación Física (Sánchez-Oliva et al., 2012) al contexto deportivo, los resultados obtenidos demuestran que el instrumento registra unas propiedades psicométricas adecuadas. Se analizaron dos modelos de medida. En primer lugar, se testó un modelo el que se correlacionaron cinco factores de primer orden: intrínseca, identificada, introyectada, externa y desmotivación, en el que los índices de ajuste mostraron valores aceptables $(\mathrm{Hu}$ y Bentler, 1999). En el segundo modelo, basándonos en la estructura anterior, se introdujeron dos factores de segundo orden, "motivación autónoma" y "motivación controlada", los cuales estaban explicados por la motivación inrínseca e identificada (autónoma) e introyectada y externa (controlada), en el que los índices de ajuste también mostraron valores por encima de ,90 (Hu y Bentler, 1999), pero no sobrepasaron las puntuaciones obtenidas en el modelo 1 (Hu y Bentler, 1999). Esto demuestra que la estructura factorial de los dos modelos testados mostraron valores aceptables. No obstante, el primero de ellos registró puntuaciones más cercanas a ,95 y guarda 
mayor relación con los postulados de la TAD (Deci y Ryan, 2000).

Por otro lado, la adaptación al contexto deportivo del $C M E F$ indicaba una adecuada validez factorial y aceptables índices de fiabilidad en todos sus constructos, con puntuaciones superiores a ,70 (Nunnally, 1978). Asimismo, entre las relaciones encontradas entre los factores que componen la escala, destacaron las asociaciones encontradas entre la motivación intrínseca e identificada, registrando una relación significativa. A pesar de que estos resultados están en línea con los encontrados en el instrumento desarrollado en Educación Física (CMEF: Sánchez-Oliva et al., 2012), dicha asociación no fue tan elevada. Esto puede deberse a que los deportistas entienden de forma diferente el concepto de diversión con el concepto de aprender nuevas habilidades, mientras que en el contexto educativo, para los alumnos el hecho de aprender nuevos contenidos puede ser percibido como útil para el futuro y, poder así, experimentar prácticas más satisfactorias.

Del mismo modo, estos resultados también se relacionan con los de Lonsdale et al. (2008) en la validación del $B R S Q$, en el que obtuvieron que la motivación intrínseca y la regulación identificada se relacionaban negativamente con la desmotivación. Sin embargo, los resultados difieren con los hallazgos obtenidos por estos autores en las regulaciones introyectada y externa (Lonsdale et al. 2008), las cuales se relacionaban negativamente con los tres tipos de motivación intrínseca (para experimentar, para conocer y para conseguir cosas), con la motivación intrínseca general y con la regulación identificada, mientras que en este estudio la regulación externa se relacionó positivamente con el resto de regulaciones y con la desmotivación. Una explicación a ello puede ser que existan deportistas con un perfil motivacional autónomo y controlado con relaciones positivas, ya que realizan deporte por la satisfacción y disfrute que le proporciona, pero también por motivos externos con el propósito de demostrar su potencial al resto, ganar o llegar a deportista de alto nivel, obtener reconocimiento, etc.

En línea con lo anterior, los resultados se asemejan a los de Pelletier et al. (1995). Concretamente, estos autores hallaron que en la SMS la regulación externa se relacionó positivamente con las demás regulaciones y con la desmotivación. Puede ser que un deportista que se enorgullezca por el reconocimiento de los demás y tenga una alta regulación hacia la obtención de premios, en ocasiones vaya encaminado hacia una desmotivación si no es satisfecho este reconocimiento o no se logran los objetivos relacionados con la victoria y/o el éxito, mientras que en otros deportistas el resultado pueda ser satisfactorio y por tanto una regulación externa se relacione positivamente con las regulaciones más próximas a los mayores niveles de autodeterminación. También, al igual que en el presente estudio, Pelletier et al. (1995) obtuvieron que las tres motivaciones intrínsecas se relacionaban negativamente con la desmotivación.
Asimismo, otro de los propósitos del estudio era comprobar la invarianza del modelo propuesto en función del género. En este sentido, los resultados obtenidos mostraron que el modelo factorial testado era adecuado, a pesar de que los resultados fueron significativos. Tal y como señala el estudio de Cheung y Rensvold (2002), el coeficiente chi cuadrado es susceptible al cambio cuando se trata de estudios con muchos participantes. Pero, según estos expertos psicométricos, los valores de $\Delta \mathrm{CFI}$ inferiores o iguales a ,01 pueden considerarse suficientes como para no rechazar la hipótesis y considerar al modelo invariante. En esta línea, los resultados difieren a los hallados en la versión de origen del CMEF de Sánchez-Oliva et al. (2012), en la medida de que estos estos autores no obtuvieron diferencias significativas en el coeficiente de chi cuadrado. No obstante, Lonsdale et al. (2008) obtuvieron hallazgos similares a los encontrados en el presente estudio con $\triangle \mathrm{CFI}$ inferiores o iguales a ,01. Asimismo, también registraron diferencias significativas entre el coeficiente de chi cuadrado entre el modelo sin restricciones y los diferentes modelos con invarianza.

\section{Aplicaciones prácticas}

Como posibles aplicaciones de futuro, sería interesante replicar el estudio con otra muestra formada por deportistas de edad adulta para comprobar y verificar la adecuación de la escala en otros contextos y con otras edades, incluyendo en su análisis ítems diseñados para valorar la regulación integrada. Además, puede servir para conocer el tipo de motivación de manera aplicada en cualquier contexto deportivo, independientemente de la modalidad que practiquen los adolescentes y en un rango de edad que contempla varias categorías. Por otro lado, las implicaciones prácticas irían encaminadas a seguir utilizando dicha escala en el ámbito científico, tratando de ser utilizada en estudios que pretendan valorar el tipo de motivación de jóvenes deportistas.

En definitiva, podríamos concluir con que el modelo de cinco factores correlacionados obtuvo adecuadas puntuaciones en todos los análisis estadísticos sometidos para comprometer su adaptación al contexto deportivo, erigiéndose como un modelo más fiable y válido que el de tres factores correlacionados. Por tanto, se presenta una escala con adecuadas propiedades psicométricas que permite a la comunidad científica valorar los tipos de regulaciones motivacionales de jóvenes deportistas.

Agradecimientos. Este trabajo ha sido realizado gracias a la financiación y colaboración del Fondo Social Europeo (FSE) y la Dirección General de Modernización e Innovación Tecnológica del Gobierno de Extremadura (Consejería de Empleo, Empresa e Innovación) mediante la convocatoria de «Ayudas para la Formación del Personal Investigador Predoctoral (PD12112)». 


\section{Referencias}

1. American Psychological Association (2009). Publication Manual of the American Psychological Association, Sixth Edition.

2. Bagozzi, R. P., y Kimmel, S. K. (1995). A comparison of leading theories for the prediction of goal-directed behaviours. British Journal of Social Psychology, 34(4), 437-461.

3. Balaguer, I., Castillo, I., y Duda, J. L. (2007). Propiedades psicométricas de la Escala de Motivación Deportiva en deportistas españoles. Revista Mexicana de Psicología, 24(2), 197-207.

4. Barkoukis, V., y Hagger, M. S. (2013). The trans-contextual model: perceived learning and performance motivational climates as analogues of perceived autonomy support. European Journal of Psychology of Education, 28(2), 353-372.

5. Brière, N. M., Vallerand, R. J., Blais, M. R., y Pelletier, L. G. (1995). Développement et Validation d'une Mesure de Motivation Intrinsèque, Extrinsèque et d'Amotivation en Contexte Sportif: L'Échelle de Motivation dans les Sports (ÉMS). International Journal of Sport Psychology, 26(4), 465-489.

6. Byrne, B. M. (2008). Testing for multigroup equivalence of a measuring instrument: A walk through the process. Psichothema, 20, 872 882.

7. Cheung, G. W., y Rensvold, R. B. (2002). Evaluating goodness of fit indexes testing measurement invariance. Structural Equation Modeling, 9, 233-255.

8. Deci, E. L., y Ryan, R. M. (1985). Intrinsic motivation and self-determination in human behavior. New York: Plenum Press.

9. Deci, E. L., y Ryan, R. M. (2000). Target Article: The "What" and "Why" of Goal Pursuits: Human Needs and the Self-Determination of Behavior. Psychological Inquiry, 11(4), 227-268.

10. Guzmán, J. F., Carratalá, E., García-Ferriol, A., y Carratalá, V. (2010). Propiedades psicométricas de una escala de motivación deportiva. $M o$ tricidad. European Journal of Human Movement, 16, 85-98.

11. Hendry, D. T., Crocker, P. R., y Hodges, N. J. (2014). Practice and play as determinants of self-determined motivation in youth soccer players. Journal of Sports Sciences, 32(11), 1091-1099.

12. Hu, L., y Bentler, P. M. (1999). Cutoff criteria for fit indexes in covariance structure analysis: Conventional criteria versus new alternatives. Structural Equation Modeling, 6, 1-55.

13. Lonsdale, C., Hodge, K., y Rose, E. A. (2008). The behavioral regulation in sport questionnaire (BRSQ): instrument development and initial validity evidence. Journal of Sport and Exercise Psychology, 30(3), 323-355.

14. Lonsdale, C., Hodge, K., Hargreaves, E. A., y Ng, J. Y. (2014). Comparing sport motivation scales: A response to Pelletier et al. Psychology of Sport and Exercise, 15(5), 446-452.

15. López, J. M. (2000). Estandarización de la Escala de Motivación en el Deporte (EMD) de Brière N. M., Vallerand, R. J., Blais, M. R. y Pelletier, L. G., en deportistas mexicanos. Motricidad. European Journal of Human Movement, 6, 67-93.

16. McDonald, R.P. (1985). Factor analysis and related methods. Hillsdale NJ: Erlhaum.

17. McDonald, R.P (1999). Test theory. A unified treatment. Mahwah, NJ, Lawrence Erlbaum Associates.

18. Mallett, C., Kawabata, M., Newcombe, P., Otero-Forero, A., y Jackson, S. (2007). Sport Motivation Scale-6 (SMS-6): A revised six-factor sport motivation scale. Psychology of Sport and Exercise, 8, 600-614.

19. Mallett, C., Kawabata, M., y Newcombe, P. (2007). Progressing measurement in sport motivation with the SMS-6: A response to Pelletier, Vallerand, and Sarrazin. Psychology of Sport and Exercise, 8, 622-631.

20. Marsh, H. W., Hau, K-T., y Grayson, D. (2005). Goodness of fi t evaluation in structural equation modeling. En A. Maydeu-Olivares y J.
McCardle (Eds.): Contemporary psychometrics: A Festschrift to Roderick P. Mc-Donald (pp. 275-340). Mahwah, NJ: Erlbaum.

21. Martin, J. J., y Cutler, K. (2002). An exploratory study of flow and motivation in theater actors. Journal of Applied Sport Psychology, 14, 344-352.

22. Moran, G. S., Russinova, Z., Yim, J. Y., y Sprague, C. (2013). Motivations of Persons with Psychiatric Disabilities to Work in Mental Health Peer Services: A Qualitative Study Using Self-Determination Theory. Journal of Occupational Rehabilitation, 34(1), 32-41.

23. Moreno-Murcia, J. A., Marzo, J. C., Martínez-Galindo, C., y Conte, L. (2011). Validación de la Escala de "Satisfacción de las Necesidades Psicológicas Básicas” y del Cuestionario de la "Regulación Conductual en el Deporte" al contexto español. Revista Internacional de Ciencias del Deporte, 7(26), 355-369.

24. Nunnally, J. C. (1978). Psychometric theory. New York: McGraw-Hill Inc.

25. Nunnally, J. C., y Bernstein, I. H. (1994). Psychometric theory (3rd ed.). New York, NY: McGraw-Hill.

26. Núñez, J. L., Martín-Albo, J., y Navarro, J. (2007). Propiedades psicométricas de la versión española de la escala de motivación deportiva. Revista de Psicología del Deporte, 16(2), 211-223.

27. Pelletier, L. G., Fortier, M. S., Vallerand, R. J., Tuson, K. M., Briere, N. M., y Blais, M. R. (1995). Toward a new measure of intrinsic motivation, extrinsic motivation, and amotivation in sports: The Sport Motivation Scale (SMS). Journal of Sport and Exercise Psychology, 17, 35-53.

28. Pelletier, L. G., Vallerand, R. J., y Sarrazin, P. (2007). The revised six-factor Sport Motivation Scale (Mallett, Kawabata, Newcombe, Otero-Forero, y Jackson, 2007): Something old, something new, and something borrowed. Psychology of Sport and Exercise, 8, 615-621.

29. Pelletier, L. G., Rocchi, M. A., Vallerand, R. J., Deci, E. L., y Ryan, R. M. (2013). Validation of the revised sport motivation scale (SMS II). Psychology of Sport and Exercise, 14(3), 329-341.

30. Rotter, J. B. (1966). Generalized expectancies for internal versus external control of reinforcement. Psychological Monographs, 80, Whole No. 609.

31. Ryan R. M., y Deci, E. L. (2000). Self-determination theory and the facilitation of intrinsic motivation, social development and wellbeing. American Psychologist, 55(1), 68-78.

32. Sánchez-Oliva, D., Leo, F. M, Amado, D., González-Ponce, I., y García-Calvo, T. (2012). Desarrollo de un cuestionario para valorar la motivación en educación física. Revista Iberoamericana de Psicología del Ejercicio y Deporte, 7(2), 227-250.

33. Sánchez, J. M., y Núñez, J. L. (2007). Análisis preliminar de las propiedades psicométricas de la versión espańola de la Escala de Necesidades Psicológicas Básicas en el Ejercicio Físico. Revista Iberoamericana de Psicología del Ejercicio y el Deporte, 2(2), 83-92.

34. Vallerand, R. J., y Rousseau, F. L. (2001). Intrinsic and extrinsic motivation in sport and exercise: A review using the hierarchical model of intrinsic and extrinsic motivation. En R. N. Singer, H. A. Hausenblas y C. M. Janelle (Eds.): Handbook of sport psychology (2a ed., pp. 389416). New York: John Wiley and Sons.

35. Vallerand, R. J. (2007). Intrinsic and Extrinsic Motivation in Sport and Physical Activity. En G. Tenenbaum y R. C. Eklund (Eds.), Handbook of sport Psychology (3a ed., pp. 59-83). New York: Wiley.

36. Vansteenkiste, M., Lens, W., y Deci, E. L. (2006). Intrinsic versus extrinsic goal contents in self-determination theory: Another look at the quality of academic motivation. Educational Psychologist, 41, 19-31.

37. Vansteenkiste, M., Niemiec, C. P., y Soenens, B. (2010). The development of the five mini-theories of self-determination theory: an histo- 
rical overview, emerging trends, and future directions, in Timothy C. Urdan, Stuart A. Karabenick (ed.) The Decade Ahead: Theoretical Perspectives on Motivation and Achievement (Advances in Motivation and Achievement, Volume 16), Emerald Group Publishing Limited, pp.105-165.

38. Viladrich, C., Torregrosa, M., y Cruz, J. (2011). Calidad psicométrica de la adaptación española del Cuestionario de Regulación Conductual en el Deporte. Psicothema, 23(4), 786-794.

39. Vlachopoulos, S. P., Karageorghis, C. I., y Terry, P. C. (2000). Motivation profiles in sport: A self-determination theory perspective. Research Quarterly for Exercise and Sport, 71, 387-397.

\section{Anexo 1. Cuestionario de Motivación en el Deporte (CMD)}

Yo practico deporte...

- Intrínseca:

1.- Porque es divertido.

6.- Porque me resulta agradable e interesante.

11.- Porque me lo paso bien realizando los ejercicios.

16.- Por la satisfacción que siento al practicarlo.

- Identificada:

2.- Porque puedo aprender habilidades que podría usar en otras áreas de mi vida.

7.- Porque valoro los beneficios que puede tener para desarrollarme como persona.

12.- Porque, para mí, es una de las mejores formas de conseguir capacidades útiles para mi futuro.

17.-Porque me aporta conocimientos y habilidades que considero importantes.

- Introyectada:

3.- Porque es lo que debo hacer para no sentirme mal conmigo mismo.

8.- Porque me siento mal si no lo hago.

13.- Porque lo veo necesario para no sentirme mal conmigo mismo.

18.- Porque me siento mal conmigo mismo si falto a los entrenamientos.

\section{- Externa:}

4.- Porque está bien visto por el entrenador y los compañeros.

9.- Porque quiero que los demás piensen que soy un buen deportista.

14.- Porque quiero que mis compañeros valoren lo que hago.

19.- Para demostrarles al entrenador y mis compañeros mi interés.

- Desmotivación:

5.- Pero no comprendo por qué practico deporte.

10.- Pero realmente siento que estoy perdiendo mi tiempo.

15.- No lo sé, tengo la impresión de que es inútil seguir practicándolo.

20.- No lo sé claramente, porque no me gusta nada. 